\title{
An Evaluation of Methods and Available Software for Seismic Refraction Tomography Analysis
}

\author{
Jacob R. Sheehan ${ }^{1}$, William E. Doll ${ }^{1}$ and Wayne A. Mandell ${ }^{2}$ \\ ${ }^{1}$ Oak Ridge National Laboratory, Oak Ridge, TN 37831 \\ ${ }^{2}$ United States Army Environmental Center, Aberdeen, MD 21010
}

\begin{abstract}
Seismic refraction tomography is an alternative to conventional seismic refraction analysis methods. While the limitations and potential pitfalls of conventional refraction methods are wellknown the same is not true for refraction tomography. As refraction tomography becomes more widely used, the need to know and understand its capabilities as well as its limitations becomes more critical. In this study we created eight representative models for use in evaluating three commercially available codes as well as refraction tomography in general. These models range from simple two-layer or dipping-layer problems to more complicated models designed to represent features of karst terrains. We demonstrate quantitatively and qualitatively that all three codes perform at a similar level, although each has strengths and weaknesses. Refraction tomography performs well in many situations where conventional methods fail, e.g., where lateral or vertical gradients compose a significant component of the velocity structure.
\end{abstract}

\section{Introduction}

Conventional delay time or generalized reciprocal method refraction analyses make simplifying assumptions about the velocity structure that conflict with frequentlyobserved near-surface attributes, such as heterogeneity, lateral discontinuities, and gradients. Relative benefits of conventional approaches are discussed elsewhere (e.g., Lankston, 1990; Palmer, 1980). Here, we examine an alternative approach, refraction tomography, which is not subject to these constraints. Refraction tomography is able to resolve velocity gradients and lateral velocity changes and may be applied in settings where conventional refraction techniques fail, such as areas of compaction, karst, and fault zones (Zhang and Toksoz, 1998). In this paper we examine and discuss three commercial refraction tomography codes, Rayfract ${ }^{\mathrm{TM}}$ (version 2.51) (Intelligent Resources Inc), GeoCT-II (version 2.3) (GeoTomo, LLC), and SeisImager/2D (version 2.62) (OYO). Each has its own theoretical foundation with associated strengths and weaknesses, and these are assessed along with the overall performance of refraction tomography. We also briefly compare some delay-time results to the tomography results in order to demonstrate when tomography might give improved results and when it might not.

The long-term goal of this study has been to assess the performance of these codes for karst terrains, as these are particularly problematic for conventional seismic refraction analysis methods. These terrains frequently contain sinkholes, irregular and gradational bedrock interfaces, remnants of high velocity bedrock above these interfaces, deeply weathered fractures and voids that may be air-, water-, or mud-filled. All of these conditions are addressed inadequately with conventional delay-time or generalized reciprocal techniques. These methods do not take into account lateral discontinuity, propensity for gradients, and velocity inversions.

Our assessment could not, however, begin with complicated karst models, as it was necessary to first address more fundamental models. As such, this paper is primarily an evaluation of the performance of refraction tomography methods in a general sense, with specific comments on three currently available commercial software products. The analysis progresses from simple models to more complicated karst-like models.

Preliminary results from this study were presented earlier (Sheehan et al., 2003). The results presented here vary somewhat from the results presented earlier, reflecting ongoing product development by the software vendors. The code development has been ongoing throughout this study, and undoubtedly will continue in the future. Therefore even these results should not be thought of as the final word on the specific aspects and capabilities of the codes themselves. Instead this should be seen as a report on the current state of refraction tomography and of some refraction tomography codes.

\section{Processing Flow and Functionality}

Each of the three codes has its own distinct analysis flow and techniques. Not all features of the codes are 
Journal of Environmental and Engineering Geophysics

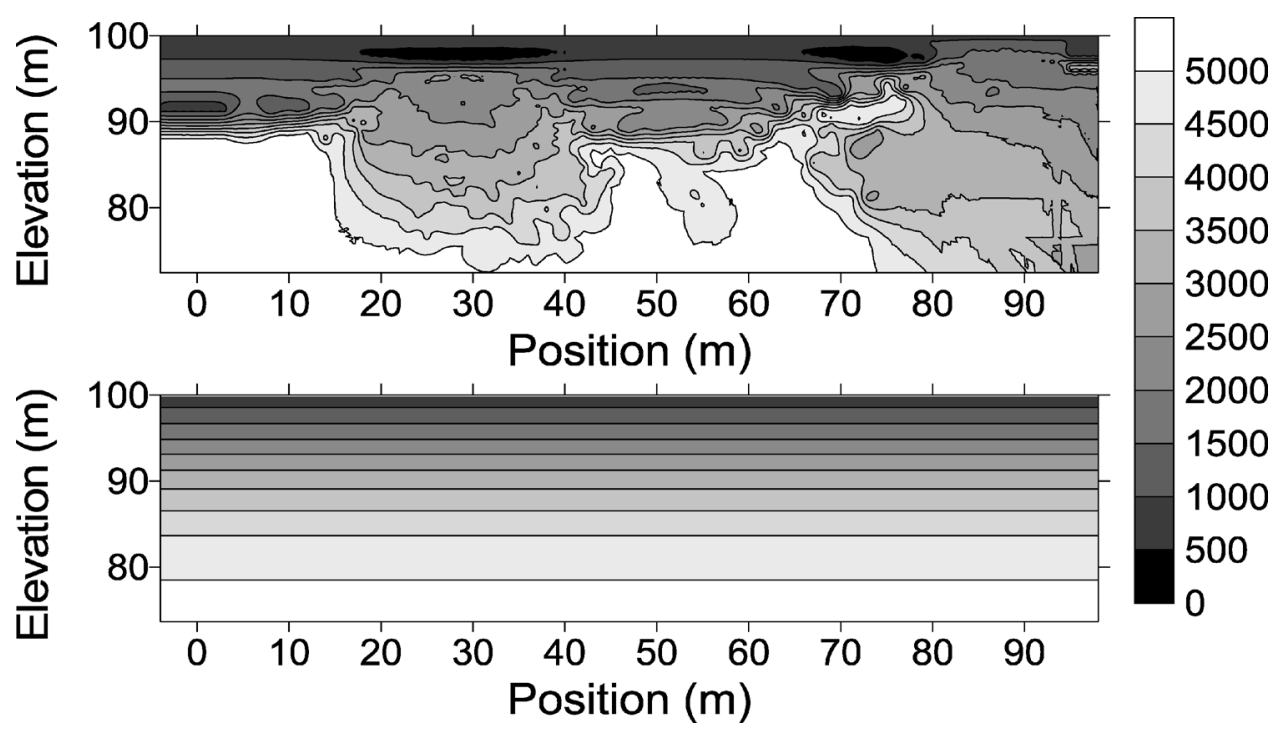

Figure 1. Delta-t-V result (top) and simple gradient model generated from it (bottom) for velocity (m/s) Model 6 (Fig. 5, discussed later).

discussed here because the emphasis of this study is on the tomography algorithms contained in each code. All three codes incorporate inversion techniques other than tomography that have not been explored. In addition, all three codes have a first arrival picking capability that was not used for this study and is not discussed here.

\section{Rayfract}

The processing flow for Rayfract is as follows. First a database is created for the traveltime data and geometry to be stored. Next the data are imported into the database. Once the data are imported, the survey geometry must either be imported or specified from within the code. Once the data is imported and geometry specified there are two processing options. The difference between the two options is in what initial model is used.

The first option is to use the Delta-t-V method (Gebrande and Miller, 1985; Rohdewald, 1999; and Rohdewald, in preparation) to generate an initial model. This result can then be gridded using Surfer or other gridding software. Rayfract can automatically link to Surfer 8 and grid this file for the user, or it can be done manually using Surfer or another gridding software package. Manual gridding allows control over the grid spacing, which determines the grid spacing used for the tomographic analysis. Using the Delta-t- $\mathrm{V}$ method helps identify small features and velocity inversions. The disadvantage of using the Delta-t- $\mathrm{V}$ output for the initial model is that there may be artifacts in the Delta-t- $\mathrm{V}$ output that are not removed completely by the subsequent tomography algorithm.

The second option within Rayfract is to use the "smooth inversion" algorithm. This automatically creates a one dimensional initial model based on the Delta-t-V results. It then extends this $1 \mathrm{D}$ model to cover the $2 \mathrm{D}$ area of study. Figure 1 shows a Delta-t-V output and the resulting initial model. Because it starts with a simple smooth model, there are no artifacts of concern in the initial model. The default settings for tomography using either method usually works well, although a second run with less smoothing may help bring out detail missed with the default smoothing.

\section{$\underline{\text { SeisImager }}$}

The processing flow for SeisImager is simpler than for Rayfract. There is no database as with Rayfract. Instead the data are simply imported into the code. A topography file can be imported, but is not necessary for analysis. Without a topography file, a flat surface is selected at a specified elevation. There are two ways of creating the initial model. The first is to use a time-term inversion algorithm contained in the code to generate a simple layered model. This layered model is then converted to a grid model. This is a useful method for simple data where distinct slopes can be associated with particular refractors. The alternative is to create a pseudogradient model by specifying a velocity range, dimensions and number of layers. Unlike Rayfract, where the grid spacing of the initial model can be set by the user and controls the grid spacing of the tomography, there is no control over the grid spacing in SeisImager. Instead the code creates a variable cell size with smaller cells at the surface and larger cells deeper in the model. This is presumably to decrease the processing time and to avoid artifacts caused by low ray coverage at greater depths. The default settings usually work well, although, like with Rayfract, a second run with less smoothing can help bring out smaller features. 
Sheehan et al.: Seismic Refraction Tomography Analysis

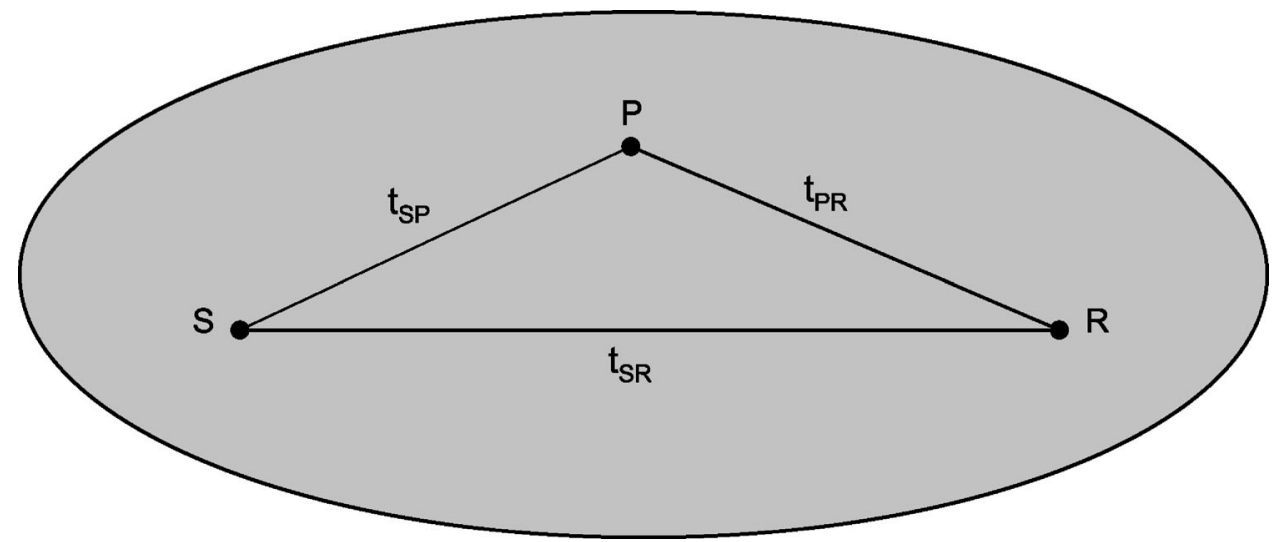

Figure 2. Schematic showing the Fresnel volume approach.

\section{GeoCT-II}

GeoCT-II is derived from much more costly codes developed for the petroleum industry. GeoCT-II is unique in that it uses a modular approach. A menu of subroutines opens when the code is initiated. The first step is to import the traveltimes using the first-arrival picking subroutine. Then the initial model can be generated using one of two methods. The first is to generate a model of your choosing using the model builder subroutine. This works well if you want to use a simple gradient model, as we did in this study, or you have an estimate of the velocity structure. The other method is to use a subroutine that displays the traveltime data and creates a simple layered model from picking turning points (i.e., changes in slope) in the data, displayed in a time verses offset plot. As with Rayfract, the grid spacing can be set for the initial model and is used for the tomography analysis step. Unlike the other two codes, the default settings for the tomography analysis do not work well in GeoCT-II. The default smoothing parameter is about 10 times larger than what we have found to be optimal for near-surface work. There is also an $x / y$ smoothing parameter that can be adjusted to improve the resolution and quality of the result. Fortunately, the tomography algorithm in this code is very fast (see performance comparison later in this paper) allowing quick and easy experimentation to determine the optimal settings.

\section{Analysis Techniques}

Rayfract uses the Wavepath Eikonal Traveltime (WET) method (Schuster and Quintus-Bosz, 1993; Woodward and Rocca, 1988). The inversion algorithm uses the Fresnel volume approach (Watanabe et al., 1999) which is an alternative to the raypath approach used by most inversion schemes. A Fresnel volume (Fig. 2) is defined by a set of waveforms that arrive within a half period of the fastest waveform. A point $\mathrm{P}$ is in the volume if and only if:

$$
\mathrm{t}_{\mathrm{SP}}+\mathrm{t}_{\mathrm{PR}}-\mathrm{t}_{\mathrm{SR}} \leq \frac{1}{2 f}
$$

where $t_{i j}$ refers to the traveltime for the path from point $i$ to point $\mathrm{j}$. $\mathrm{S}$ is the starting point and $\mathrm{R}$ is the end point. The methods that use ray paths make the assumption that the frequency of the source is infinite, and hence the wavelength is zero. This means that only the properties of the portions of the model through which the rays pass directly affect the results. This assumption is not accurate in reality because real waves have a finite wavelength and will be affected by adjacent parts of the model in the vicinity of the ray path. The Fresnel volume approach addresses this issue by taking into account ray paths with slightly longer travel times.

SeisImager and GeoCT-II both use a nonlinear least squares approach for the inversion step and wavefront propagation methods for the travel time modeling (Geometrics and OYO, 2003 and Zhang and Toksoz, 1998). SeisImager inverts for each traveltime individually while GeoCT-II inverts for average slowness (traveltimes divided by ray path) and apparent slowness (derivative with respect to distance of the traveltime curve). GeoCT-II also limits the roughness of the model. According to Zhang and Toksoz (1993), this approach has the effect of inverting for traveltime curves as a whole instead of individual traveltimes and helps reduce non-uniqueness. Another benefit of this approach is that the average slowness is

Table 1. Travel time differences from analytical solution.

\begin{tabular}{lccc}
\hline \hline & Rayfract & SeisImager & GeoCT-II \\
\hline $\begin{array}{l}\text { Mean } \\
\quad \text { difference (ms) }\end{array}$ & 0.20 & 0.04 & 0.03 \\
$\begin{array}{l}\text { Max } \\
\quad \text { difference (ms) }\end{array}$ & 0.38 & 0.18 & 0.12 \\
\hline
\end{tabular}




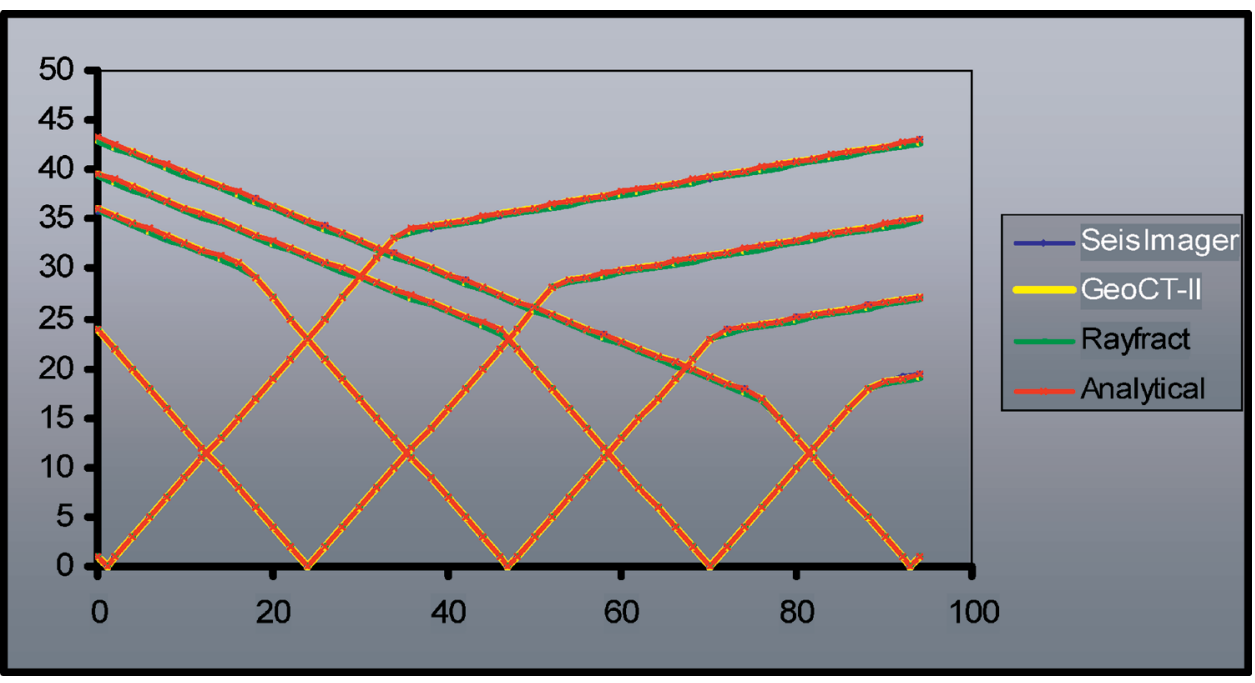

Figure 3. Modeled traveltimes, in $\mathrm{m} / \mathrm{s}$, using the three codes and a spreadsheet.

effective at finding the shallow velocity structure and the apparent slowness is better at finding the deeper structure. During the inversion the first few iterations are inverting average slowness only. Then, once the near-surface is constrained the inversion switches to a joint inversion of average slowness and apparent slowness. In this way small inaccuracies in the near-surface are less likely to lead to larger errors at depth.

The wavefront propagation method for traveltime calculation determines the time required for the wave to travel from the source to each adjacent node. The node that has the shortest traveltime path leading to it then acts as the source, and the process is repeated until the whole model is traced. (Zhang and Toksoz, 1998)

\section{Initial Models}

For results shown in this paper, the simple model that is generated for the "smooth inversion" technique was used
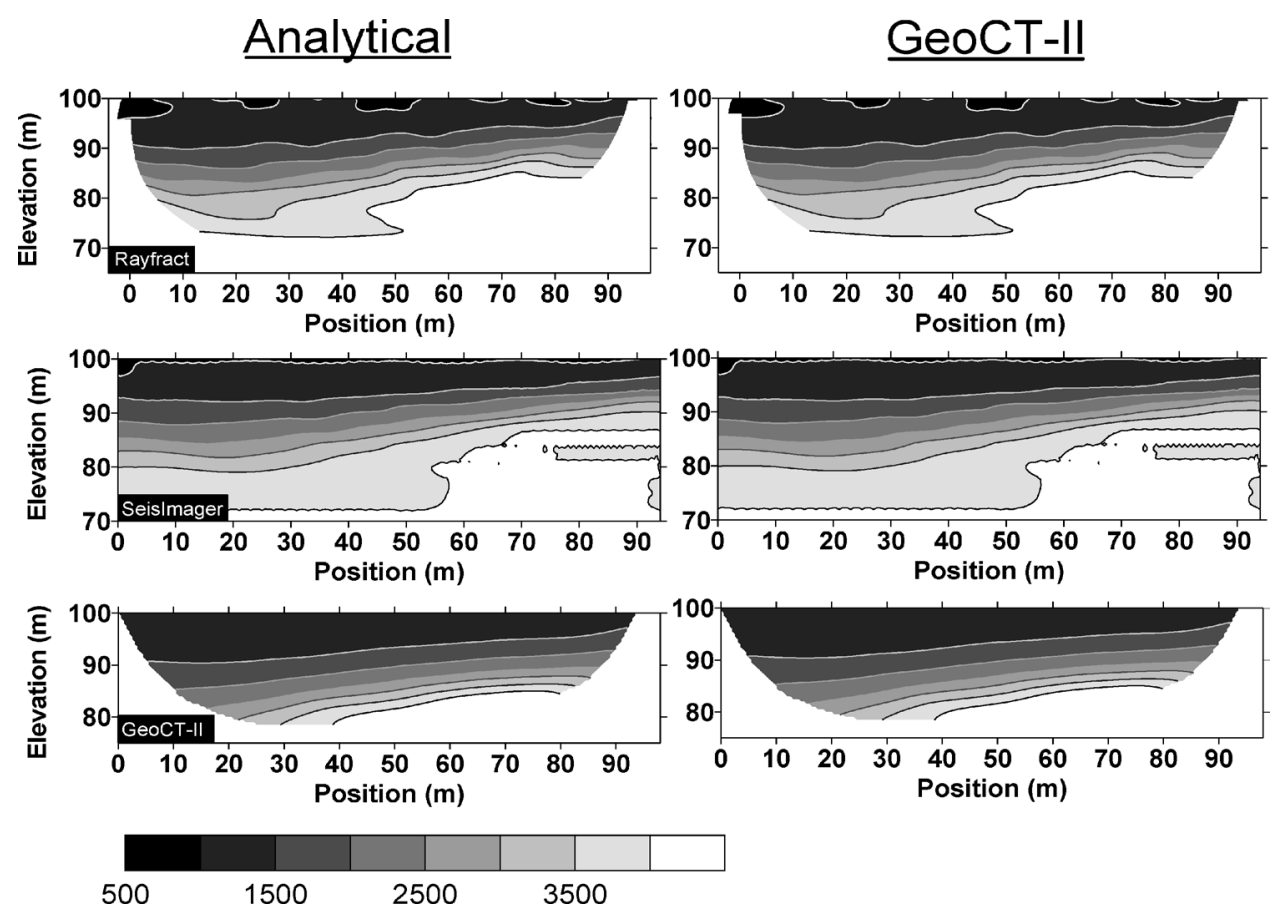

Figure 4. Comparison of tomographic velocity $(\mathrm{m} / \mathrm{s})$ results using analytical methods and GeoCT-II to generate the traveltimes. 
Sheehan et al.: Seismic Refraction Tomography Analysis
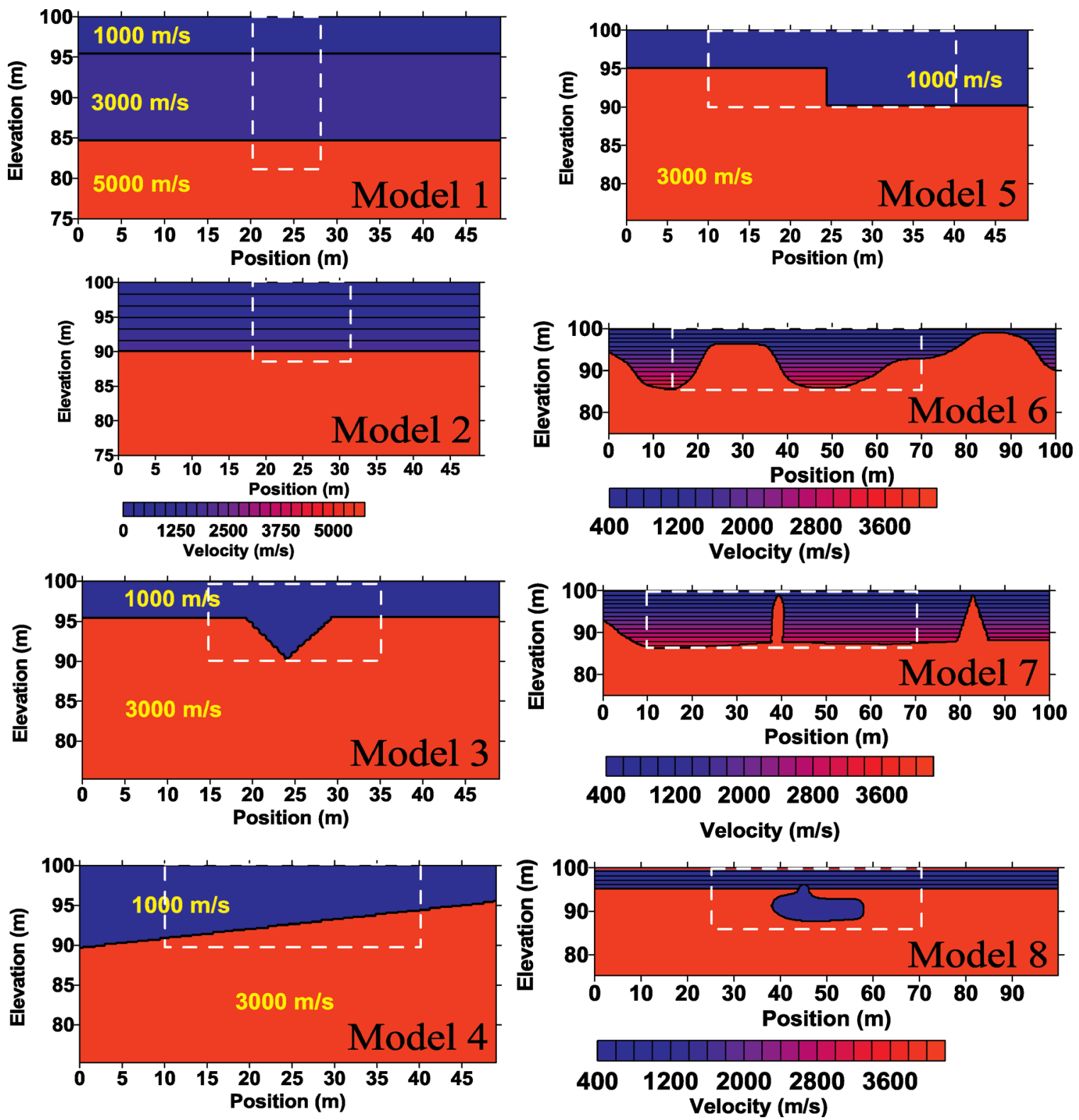

Figure 5. All velocity models used in this paper, in $\mathrm{m} / \mathrm{s}$. The white rectangles are discussed in the quantitative assessment section later in this paper.

for Rayfract as an initial model. For SeisImager and GeoCTII a gradient model was used for the initial model. For GeoCT-II there was no advantage to using the layered model generator and the tomography would sometimes fail to vary from the layered initial model returning poor results. The delay-time method in SeisImager was not used because it would do very well by itself with the simple models used here and is not helpful for the more complicated models. This would make the evaluation of simple models with SeisImager more of a test of the time-term inversion and not the tomography algorithm in SeisImager. Using the delaytime method would also void one of the advantages of tomography: reduced analysis time by elimination of the need to assign travel times to idealized refractors.

Both Rayfract and GeoCT-II use uniform cell size that is defined by the cell size of the initial model used. SeisImager uses a varying cell size that cannot be adjusted by the user. Because of this, the cell size for SeisImager is larger than the other two codes.

\section{Methodology}

Synthetic traveltimes are used for this study in order to have a true model with which to compare the tomographic 
Journal of Environmental and Engineering Geophysics

results. Because an analytical solution is only practical for the very basic models, it was necessary to use one of the codes to generate the traveltimes. Because SeisImager does not have a convenient method for generating or importing models it could not be used to generate the traveltime data sets. Rayfract can perform ray tracing on any grid file but does not have a model generator. GeoCT-II is the only code that has a convenient and robust model builder and was therefore used to generate the models. This made GeoCT-II the easiest code to use for the ray tracing because no extra file conversions were necessary. To insure that the results of this study were not biased towards GeoCT-II because it was used for the model generation, ray tracing traveltimes were calculated analytically and by using the forward modeling capability of each code for a simple dipping model. Figure 3 shows the forward modeled traveltimes from each code along with the analytical solution. The difference between the results from each code and the analytical solution is summarized in Table 1.

Because the traveltimes generated with GeoCT-II most closely match those generated independently using analytical methods there should be little or no bias towards GeoCT-II as a result of using the times generated by it. To test this, the traveltimes generated by GeoCT-II and generated analytically were both processed with all three codes. The results from this analysis (Fig. 4) are identical for each code regardless of which method was used to generate the traveltimes.

Eight synthetic models are examined in this paper (Fig. 5). They include two constant velocity layers over a half space (Model 1), gradient over a half-space (Model 2), depression (Model 3), dipping interface (Model 4), stair step (Model 5), broad epikarst (Model 6), narrow epikarst (Model 7), and cavity (Model 8). For all eight models 25 shots into 48 geophones were used in the synthetic model. As required by tomographic analysis, all 25 shots are within or immediately adjacent to the geophone spread. For the first five models the geophone spacing is one meter and for the last three it is two meters. The shot spacing is twice the geophone spacing for all models.

\section{Results}

The results of each code will be displayed together for each model. None of the codes are capable of accurately imaging abrupt velocity changes. Instead they smear the boundary into a gradient (Fig. 6). Therefore boundaries must be interpreted from areas of maximum gradient.

The results for Model 1 (constant velocity layers over half-space) are shown in Fig. 7. The first boundary is distinct in both Rayfract and SeisImager. For GeoCT-II, the boundary is more diffuse, but there is a decrease in the contour spacing at the correct location. The deeper layer boundary is inaccurate on all of the results, although it can be inferred close to the true depth on all three. This model is better suited to conventional analysis techniques than it is to tomography. The SIPT delay-time method (Haeni, et al. 1987) was able to image both transitions more accurately.

Figure 8 shows the results for Model 2 (gradient over half-space). Although all three models show a gradient, this is not meaningful because tomography will yield a gradient result even when there is no gradient. GeoCT-II shows a well-defined boundary between the gradient layer and the constant velocity half space. SeisImager also shows the boundary well but assigns a half-space velocity that is too low. Rayfract has a well-defined area of increased gradient in the vicinity of the layer boundary that covers a larger depth range than the other two results, extending the gradient further into the half-space. In order to evaluate the accuracy of the gradients modeled we calculated the vertical gradients, as shown in Fig. 9. All three codes show an area above the transition with a gradient within $25(\mathrm{~m} / \mathrm{s}) / \mathrm{m}$ of the true gradient.

For Model 3 (depression) all three results show a smoothed version of the depression with a high velocity artifact under the depression (Fig. 10). This smoothing is very pronounced in the GeoCT-II result, less pronounced in the SeisImager result and minor in the Rayfract result. Figure 11 shows the ray coverage for the GeoCT-II tomogram. Most of the artifacts are outside of the ray coverage, suggesting that the artifact is the result of the tomography algorithm updating the velocity at the center of the model only where there is adequate ray coverage.

All three results represent the dipping trend of Model 4 well (Fig. 12). SeisImager shows a few artifacts that appear raypath related. The GeoCT-II result shows an erroneous high velocity between positions 20 and 45 meters. Figure 13 shows the ray coverage. This is likely the same problem as was described above for Model 3. As with Model 1, this model is best-suited to conventional analysis methods.

The Model 5 (stair step) results show a smoothed stair step shape (Fig. 14). The Rayfract and SeisImager results show generally flat layers to the left and right of the offset, while GeoCT-II shows a dipping layer to the right of the offset. The Rayfract results show a concave feature at the step that does not exist. The SeisImager and GeoTomo results both show a drop in velocity between positions 0 and 10 meters that is caused by lack of ray coverage. Rayfract does not show this because the areas outside the ray coverage are not displayed.

All three codes do well with Model 6 (broad epikarst). They all find the general shape of the boundary, and even show the small feature located between 60 and 75 meters (Fig. 15). The only significant difference between the three codes is in the depth of the boundary. It appears to be a little 
Sheehan et al.: Seismic Refraction Tomography Analysis
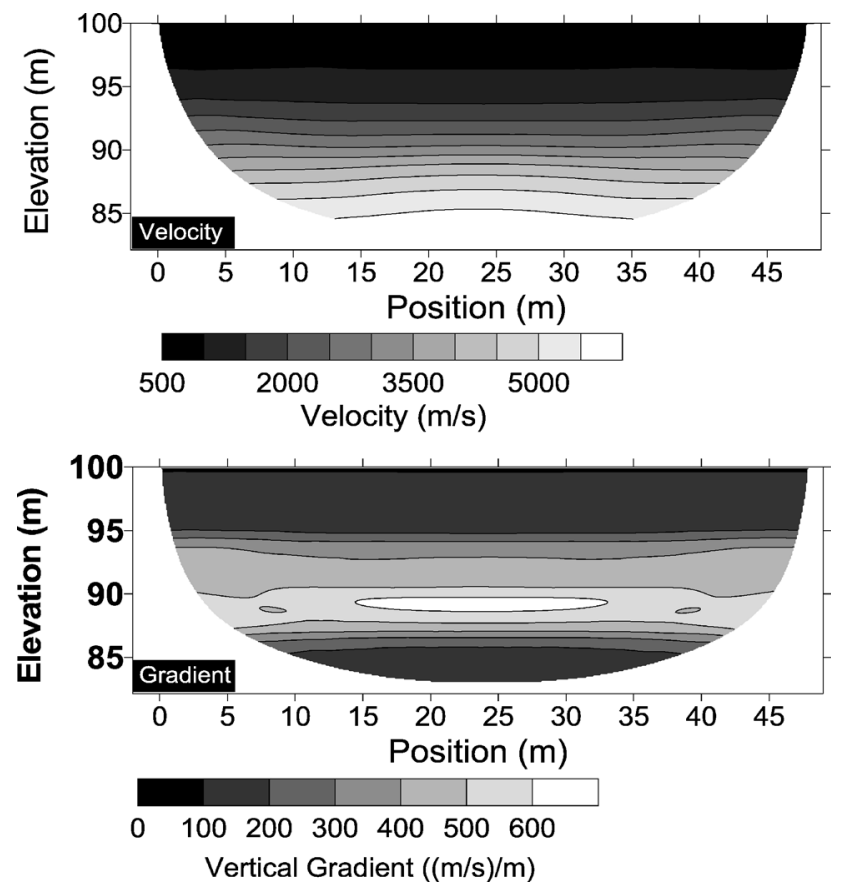

Figure 6. Demonstration of layer determination. Plots show velocity in $\mathrm{m} / \mathrm{s}$.

deeper in the GeoCT-II result than the true model or the other two results.

For Model 7 (narrow epikarst) the codes create a highly-smoothed version of the features (Fig. 16). The GeoCT-II result has high-velocity artifacts at 20 and 70 meters and a low velocity artifact at 40 meters.

The results for Model 8 (cavity) are shown in Fig. 17. None of the codes were able to properly image the cavity. SeisImager has a depression at the location of the cavity. Rayfract has a small amount of turn-in that could indicate a cavity. All three codes have an artifact that looks like a mirror image of a depression located above the feature. This is pronounced for the Rayfract and SeisImager results, and minor in the GeoCT-II result. The GeoCT-II result is very similar to the cavity, but it is too deep and located in an area of no ray coverage (Fig. 18). Unlike the other two codes GeoCT-II attempts to create features that are outside of the ray coverage. This leads to artifacts that are similar to cavities or local high velocity areas, depending on the velocity trend at the edge of the ray coverage.

\section{Efficiency}

Processing times differ among the codes. To quantify this effect, we recorded processing time for one model (Model 2) with all three codes (Table 2). We observed similar ratios of processing time for the other models. GeoCT-II runs much faster than either SeisImager or Rayfract, which both take more than twice as long as GeoCT-II. Although SeisImager and Rayfract take about
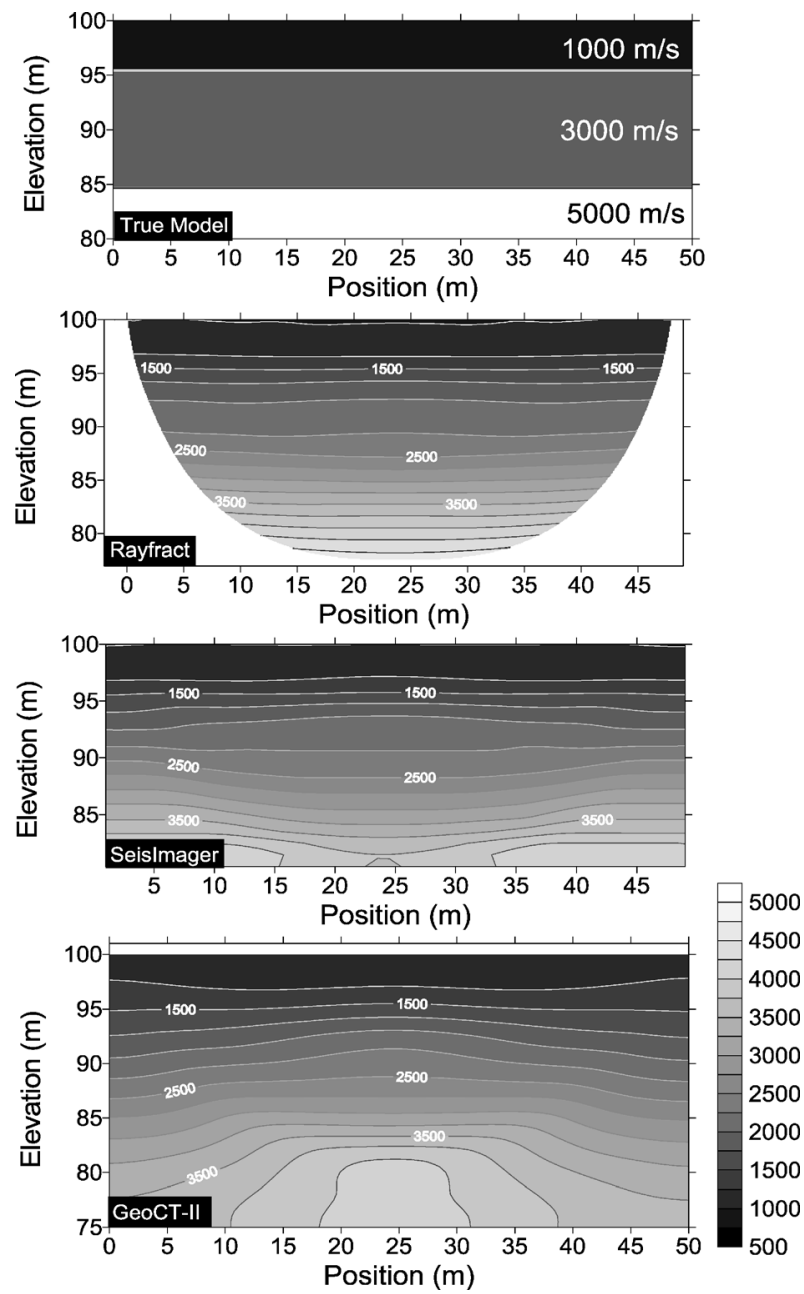

Figure 7. Velocity $(\mathrm{m} / \mathrm{s})$ results from Model 1.

equal time to run, SeisImager uses much larger cell sizes and therefore has a fraction of the total cells of either of the other codes. In the models examined in this study this large cell size has not kept SeisImager from modeling most of the features with similar accuracy to the other two codes. Note that the optimal grid spacing for GeoCT-II is 0.25 to 0.5 meters; the 0.1 meter spacing was used only for this timing analysis.

\section{Comparison of Tomography to Delay-time Method}

Although the focus of this study is refraction tomography, the models have also been analyzed in Rimrock Geophysics' SIP code which uses the delay-time method. This is done to demonstrate applications where tomography out-performs delay-time and vice-versa.

For some of the models examined in this study the delay-time method works as well or better than the tomography. The two models that demonstrate this best are models 1 and 4 (layered and dipping models). The delay- 
Journal of Environmental and Engineering Geophysics
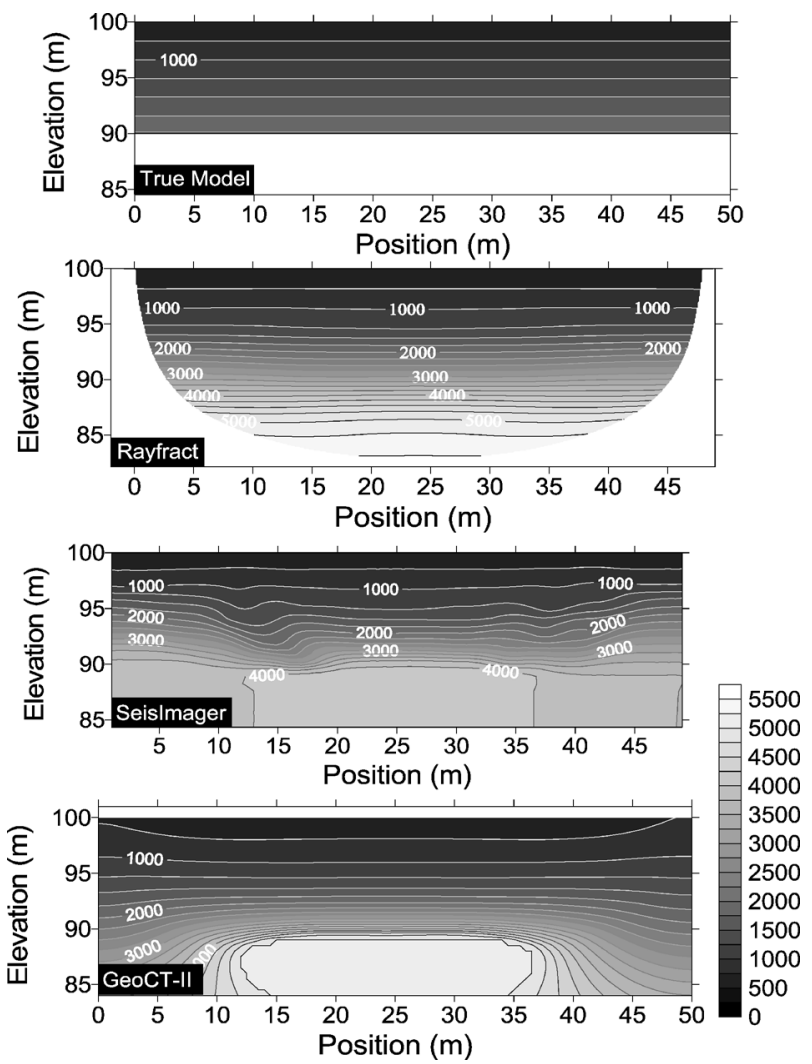

Figure 8. Velocity $(\mathrm{m} / \mathrm{s})$ results from Model 2.

time results for these two are shown in Fig. 19. Both of these models are well-suited for analysis by the delay-time method because they have simple interfaces and no gradients. While the absence of a gradient is beneficial for delay-time interpretation, it is not for tomographic analysis. As can be seen in Fig. 7 and Fig. 12, tomography properly identifies the general profile of the interface, but assigns a gradient when there is none. Because of this the delay-time method is more accurate for these two models.

For the rest of the models, tomography yields more accurate results than delay-time. Figure 20 shows the delaytime results for models 5,6 and 8 . The tomography results for these models are shown in Figs. 14, 15 and 17. For these models the tomographic results are more accurate than the delay-time results.

\section{Discussion}

Model inversion results from each code can be compared two ways, qualitatively and quantitatively. The first is by visual inspection, the second by an error calculation. The advantage of using visual inspection is that this best approximates how the results from an actual field study will be interpreted. In a field study there is no "truth" with which to compare mathematically: the result is
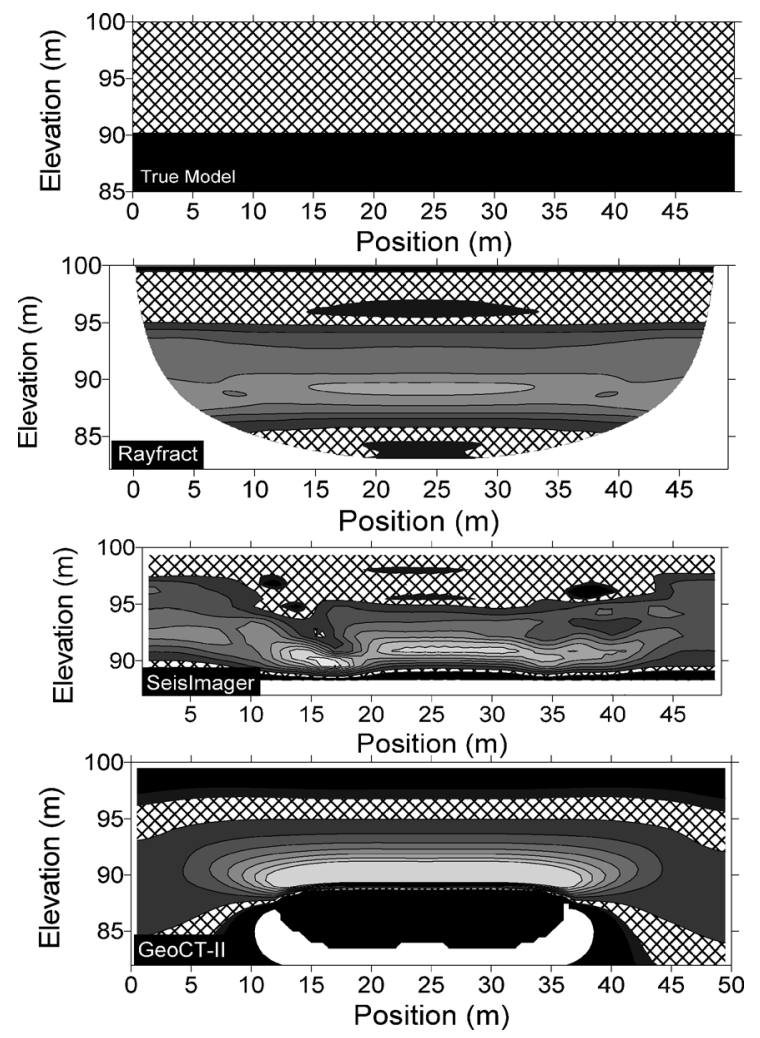

Figure 9. Vertical gradients for the Model 2 results. The areas with the pattern fill represents the true gradient of $150(\mathrm{~m} / \mathrm{s}) / \mathrm{m} \pm 25(\mathrm{~m} / \mathrm{s}) / \mathrm{m}$.

interpreted visually. On the other hand, error calculations provide a more objective means of evaluating code performance. We will take both approaches here.

Qualitative Assessment and Ranking

Of the three results for Model 1, Rayfract is most accurate. There is a small bump in the layer boundaries in all three results, but it is much less pronounced in the Rayfract result. The definition of the second layer boundary is better in GeoCT-II result, but there is also a large raypath-related artifact below the boundary.

For Model 2, the GeoCT-II result appears closer to the true model than those of either SeisImager or Rayfract. There is a sharp boundary at the correct location and no artifacts. SeisImager indicates a moderately sharp boundary, but also contains ray path related artifacts. The Rayfract result has a more diffuse boundary.

The results for Model 3 all contain an artifact directly below the depression. This artifact is more pronounced for the GeoCT-II result than the Rayfract and SeisImager results. In addition, the depression is not as well defined in the GeoCT-II result as it is in the SeisImager and Rayfract results. The artifact is less prominent in the Rayfract result than in the SeisImager result, making it more accurate. 
Sheehan et al.: Seismic Refraction Tomography Analysis
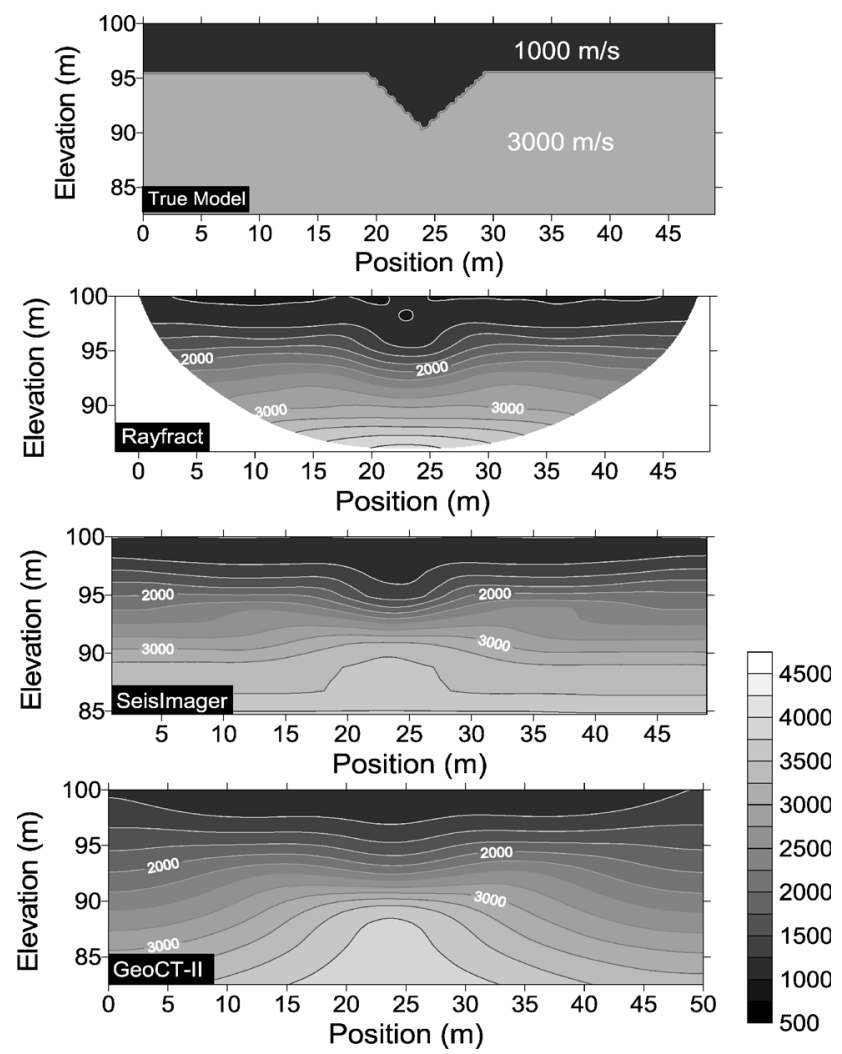

Figure 10. Velocity $(\mathrm{m} / \mathrm{s})$ results from Model 3.

The results for Model 4 all represent the dipping trend of the boundary well. Once again GeoCT-II shows ray coverage-related artifacts and SeisImager has some raypath-related artifacts. Rayfract is without significant artifacts but has a less defined boundary than the other two. Despite this Rayfract should be considered the most accurate of the three.
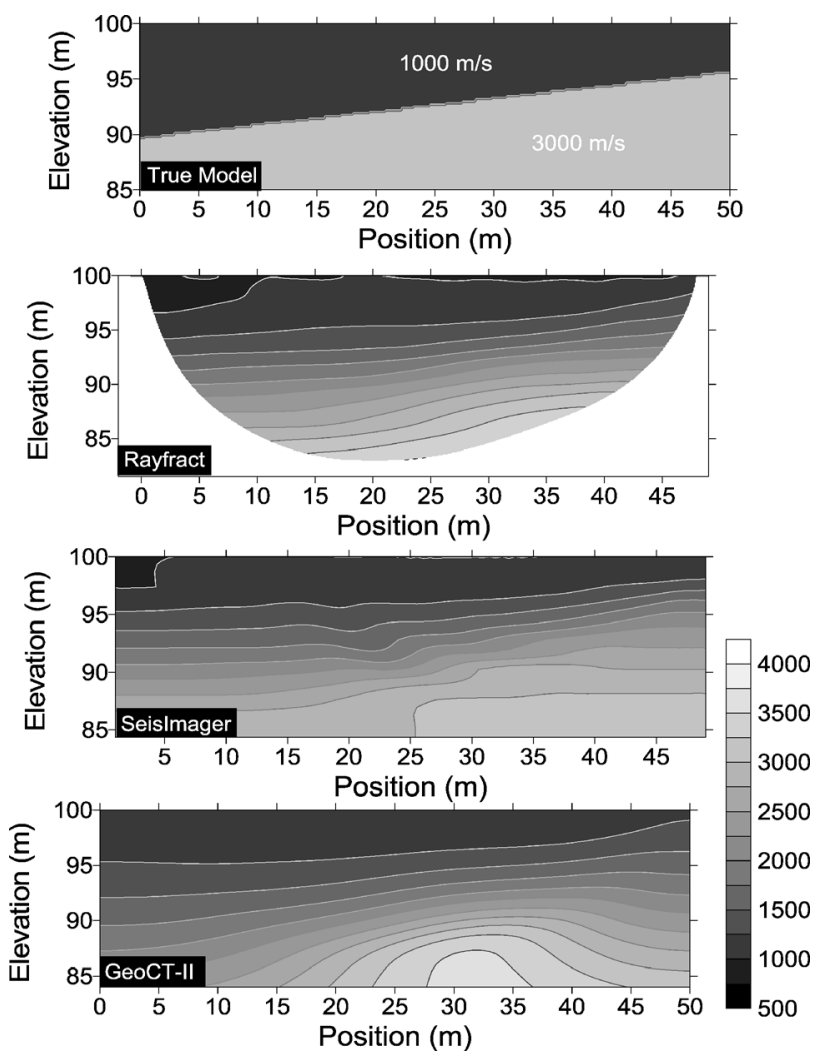

Figure 12. Velocity $(\mathrm{m} / \mathrm{s})$ results from Model 4.

All three results for Model 5 represent a smoothed version of the stair-step. GeoCT-II has a dipping boundary right of the offset, while SeisImager and Rayfract both correctly image the boundary as horizontal to the left and right of the offset. SeisImager is a little more accurate than Rayfract because the Rayfract results could lead to an interpretation of a low velocity feature underneath of stair-step.

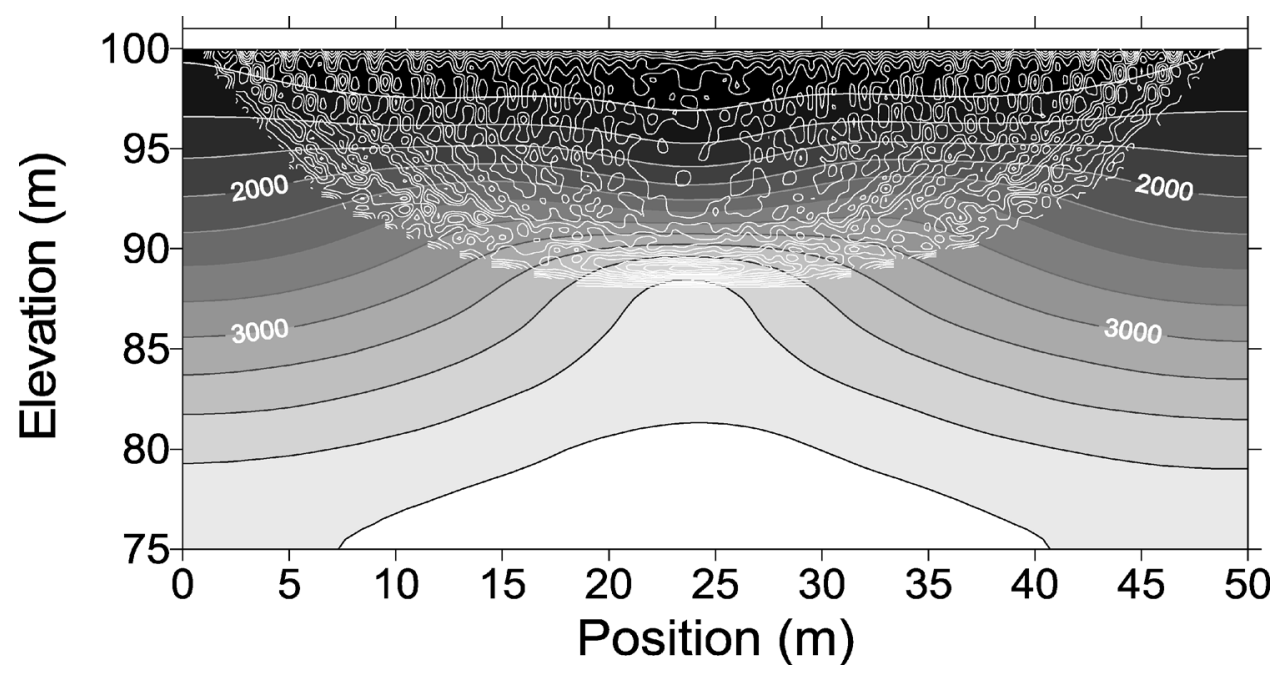

Figure 11. Ray coverage for Model 3. 
Journal of Environmental and Engineering Geophysics
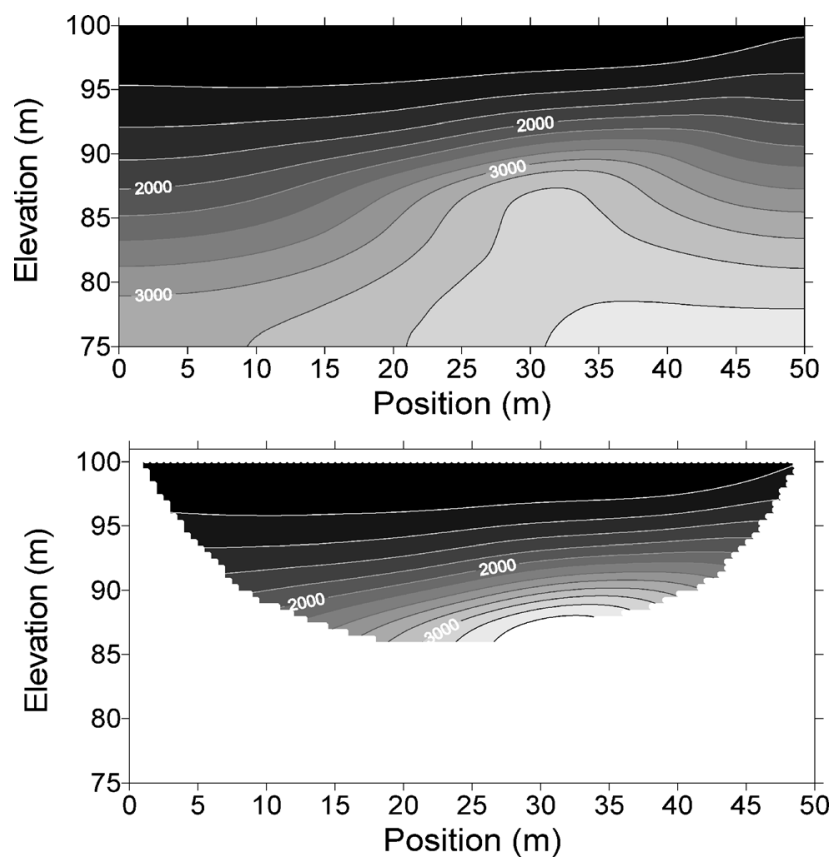

Figure 13. The top image is the full tomogram from GeoCT-II and the bottom shows only the section of the tomogram that corresponds to the area of ray coverage.
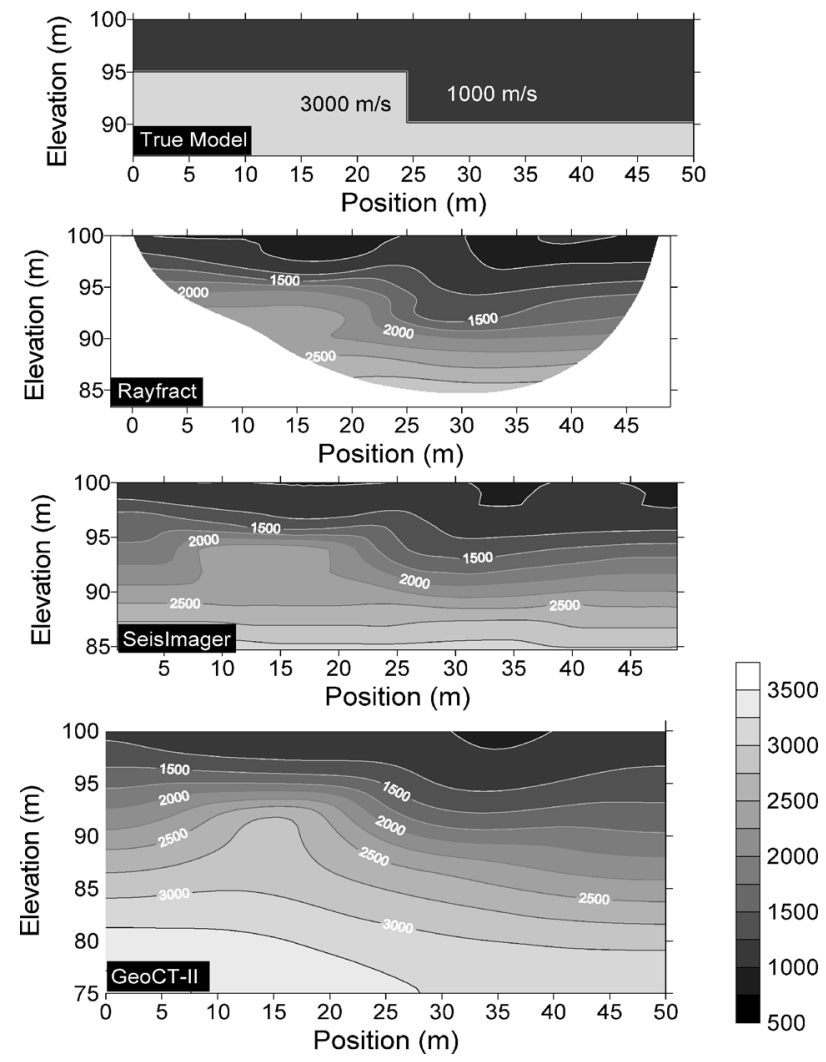

Figure 14. Velocity $(\mathrm{m} / \mathrm{s})$ results from Model 5.
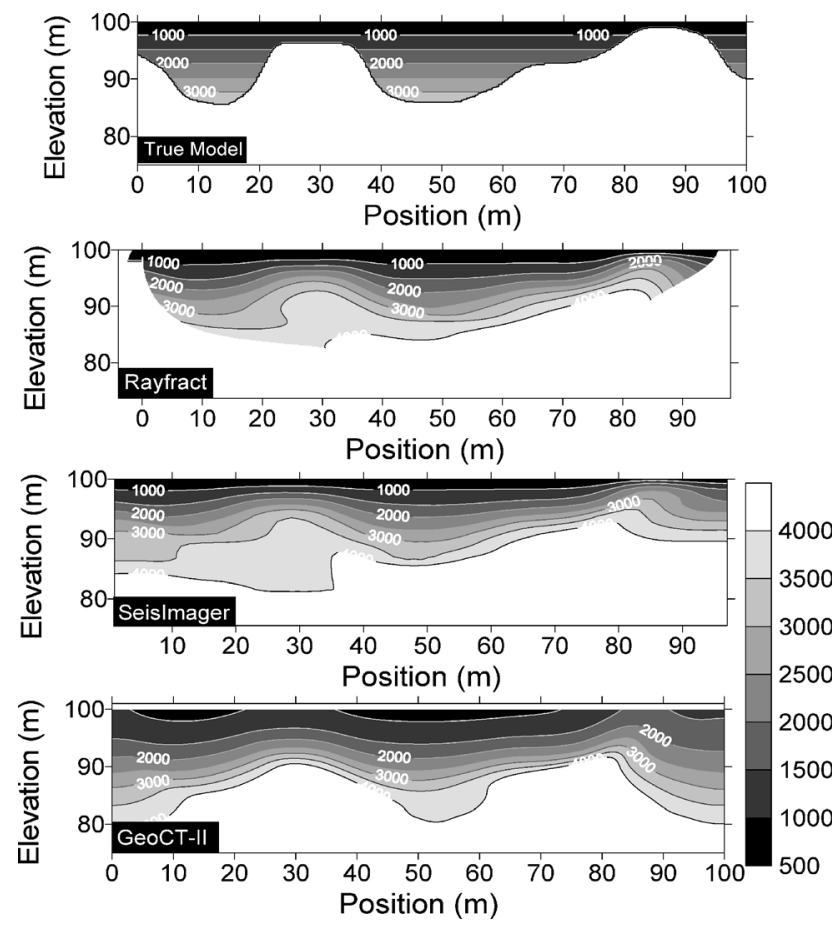

Figure 15. Velocity $(\mathrm{m} / \mathrm{s})$ results from Model 6.
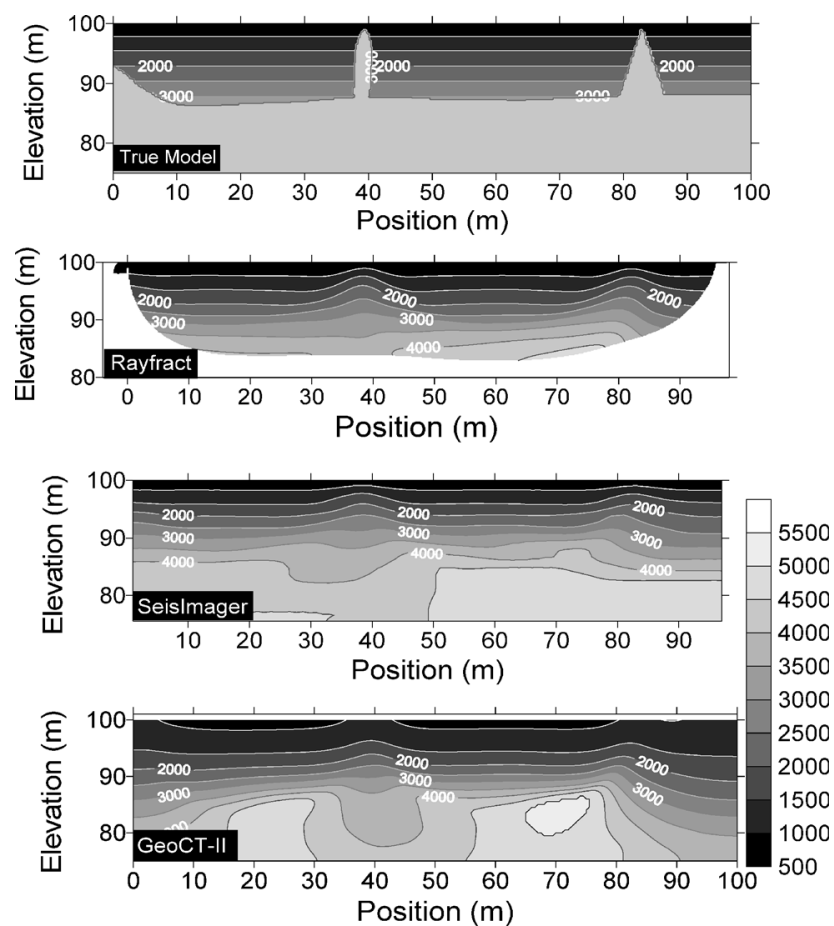

Figure 16. Velocity $(\mathrm{m} / \mathrm{s})$ results from Model 7. 

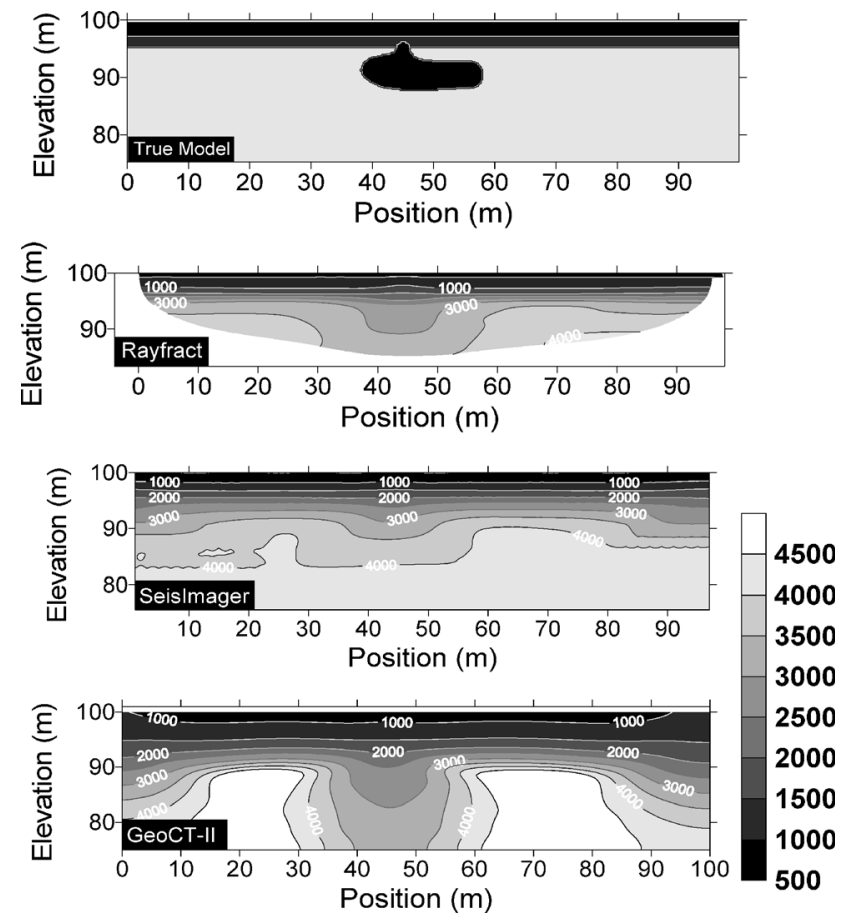

Figure 17. Velocity $(\mathrm{m} / \mathrm{s})$ results from Model 8.

Each of the codes does well with Model 6. They all capture the general shape of the boundary, and velocities are good approximations. However, GeoCT-II places the boundary too deep, making the SeisImager and Rayfract results more accurate. There is not enough difference between these two results to determine which one is better.

None of the codes could find the narrow high-velocity pinnacles in Model 7. GeoCT-II has major artifacts under the pinnacles and to the sides. The results from SeisImager and Rayfract do not contain artifacts to the extent of the GeoCTII results, although they both have some raypath related artifacts. The SeisImager and Rayfract results are both more accurate than the GeoCT-II results, and are too similar to determine which of the two best mimics the true model.
Table 2. Processing time required for each code.

\begin{tabular}{lcccc}
\hline \hline & $\begin{array}{c}\text { Grid } \\
\text { spacing } \\
(\mathbf{m})\end{array}$ & $\begin{array}{c}\text { Number } \\
\text { of cells }\end{array}$ & $\begin{array}{c}\text { Time for } \\
\mathbf{1 0} \text { iterations } \\
(\mathbf{s})\end{array}$ & $\begin{array}{c}\text { Time } \\
\text { per cell }\end{array}$ \\
\hline Rayfract & 0.1 & 130,000 & 217.8 & 0.0017 \\
SeisImager & $\sim 1^{*}$ & $816^{*}$ & 263.3 & 0.3227 \\
GeoCT-II & 0.1 & 130,000 & 94.4 & 0.0007 \\
\hline * not adjustable. & & & \\
\hline
\end{tabular}

Of the three codes, only GeoCT-II shows something that resembles a cavity for Model 8. Unfortunately, the cavity is too large, too deep and outside of the ray coverage. It is, in fact, an artifact that looks similar to the true cavity. SeisImager models the cavity the same as the depression in Model 3. Rayfract models the cavity differently than the depression, and is probably the most accurate of the three codes.

Table 3 summarizes the qualitative assessment of the codes. In cases where two results were too close to differentiate, they were each given a ranking equal to the average of the ranks not assigned to the third result.

\section{Quantitative Assessment}

The obvious disadvantage of a visual comparison is that it is subjective. Because we know the true models, we may tend to give credit for coming close visually to the true model and not consider the actual velocities in the result. To avoid this subjectivity it is necessary to use a numerical comparison. One number that can be used to compare the results is the misfit between the modeled travel-times and the true travel-times. This may be misleading because the solution to the inverse problem is non-unique. Although a model result may have lower RMS error for the modeled traveltimes than another model, it could still be further from the true velocity model.

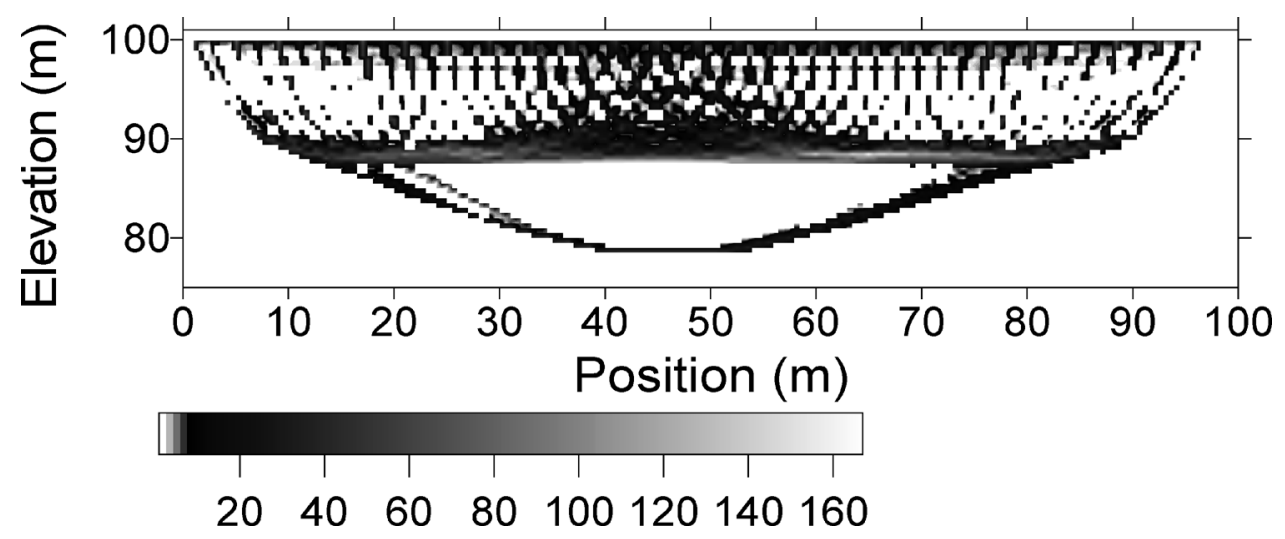

Figure 18. Model 8 ray coverage for GeoCT-II. 

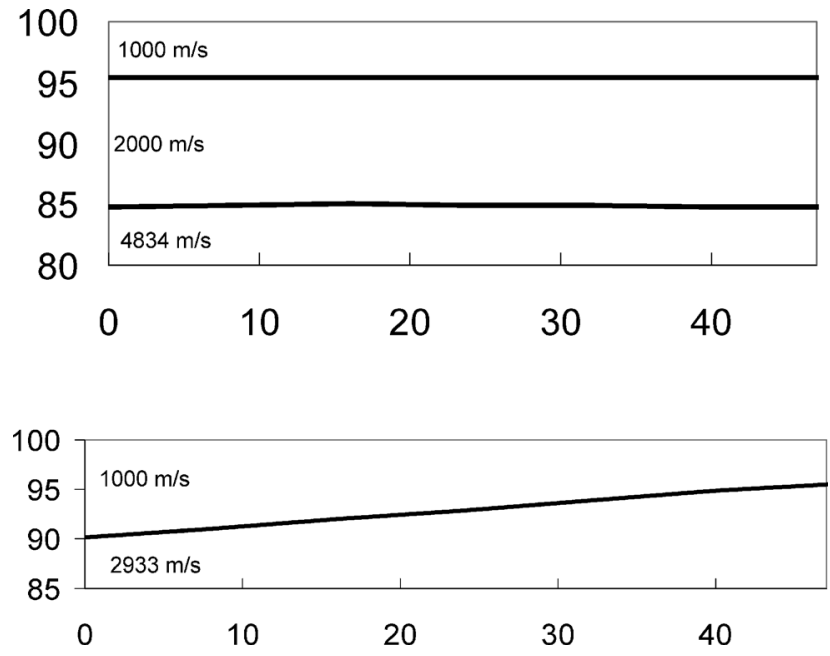

Figure 19. Delay-time results for Model 1 (top) and Model 4 (bottom).

In order to better evaluate the modeled results we have calculated RMS velocity errors for the models. Because of ray coverage differences, the models cover slightly different areas and could not be compared mathematically in their entirety. Therefore a section of each model was selected by taking the largest possible rectangle around the feature of interest while still being within the ray coverage of all three codes (see Fig. 5 for selected areas). The RMS error was calculated by comparing the calculated velocity cell-by-cell to the same area in the true model. The results from this comparison are shown in Fig. 21. The largest problem with this comparison method is that the areas of evaluation are limited. The calculated error will neglect many of the artifacts that are not near the main feature of interest.

In some cases a better fit to the true model could be
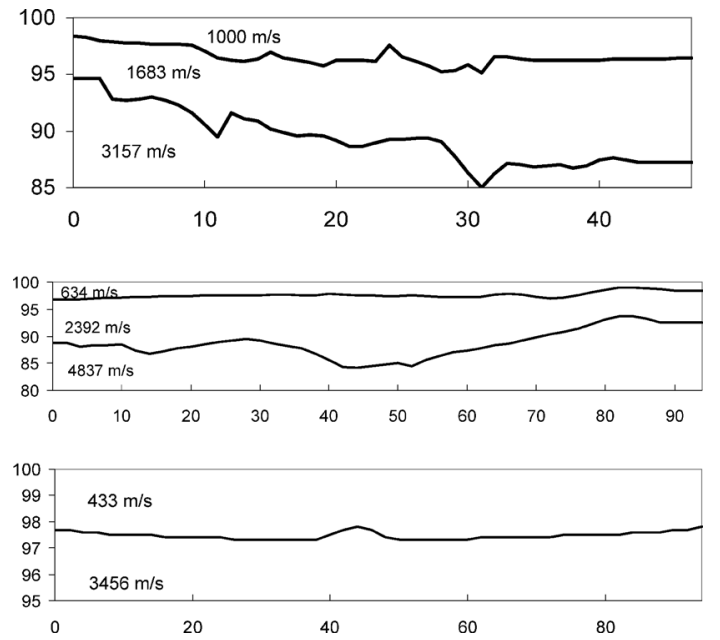

Figure 20. Delay-time results for models 5, 6 and 8 .
Table 3. Summary of qualitative assessment. Number represents the rank of the code for the particular model, with one being the best.

\begin{tabular}{lccc}
\hline \hline & Rayfract & SeisImager & GeoCT-II \\
\hline Model 1-layers & 1 & 2 & 3 \\
Model 2-gradient & 2 & 3 & 1 \\
Model 3-depression & 1 & 2 & 3 \\
Model 4-dipping & 1 & 2 & 3 \\
Model 5-stair step & 2 & 1 & 3 \\
Model 6-broad epikarst & 1.5 & 1.5 & 3 \\
Model 7-narrow epikarst & 1.5 & 1.5 & 3 \\
Model 8-cavity & 1 & 2 & 3 \\
Average Rank & 1.4 & 1.9 & 2.8 \\
\hline
\end{tabular}

obtained by using little or no smoothing. However, once noise is added to the signal, this creates results that are dominated by artifacts. A demonstration of this using GeoCT-II on Model 3 is shown in Fig. 22. Noisy data were generated by adding random errors to the pure synthetic traveltimes. For the data without random error the tomogram using minimal smoothing is a better fit to the true model. Once noise is added, the tomogram using minimal smoothing is dominated by artifacts. The tomogram using standard smoothing is the same as with the noise-free data.

\section{General Assessment}

In addition to the quality of the results, it is important to consider the functionality and flexibly of the codes. Rayfract requires a number of preliminary steps to be carried out. These steps are not difficult and may save time later, because the traveltime data, settings, and geometry are saved in the database for future analysis. The automatic "smooth inversion" analysis method is very easy to use and is the most fail-safe analysis provided by any of these codes.

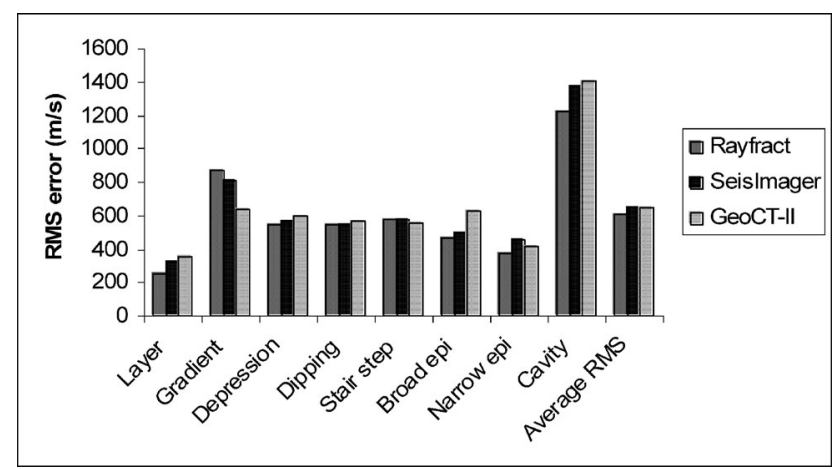

Figure 21. Velocity RMS error. 
Sheehan et al.: Seismic Refraction Tomography Analysis
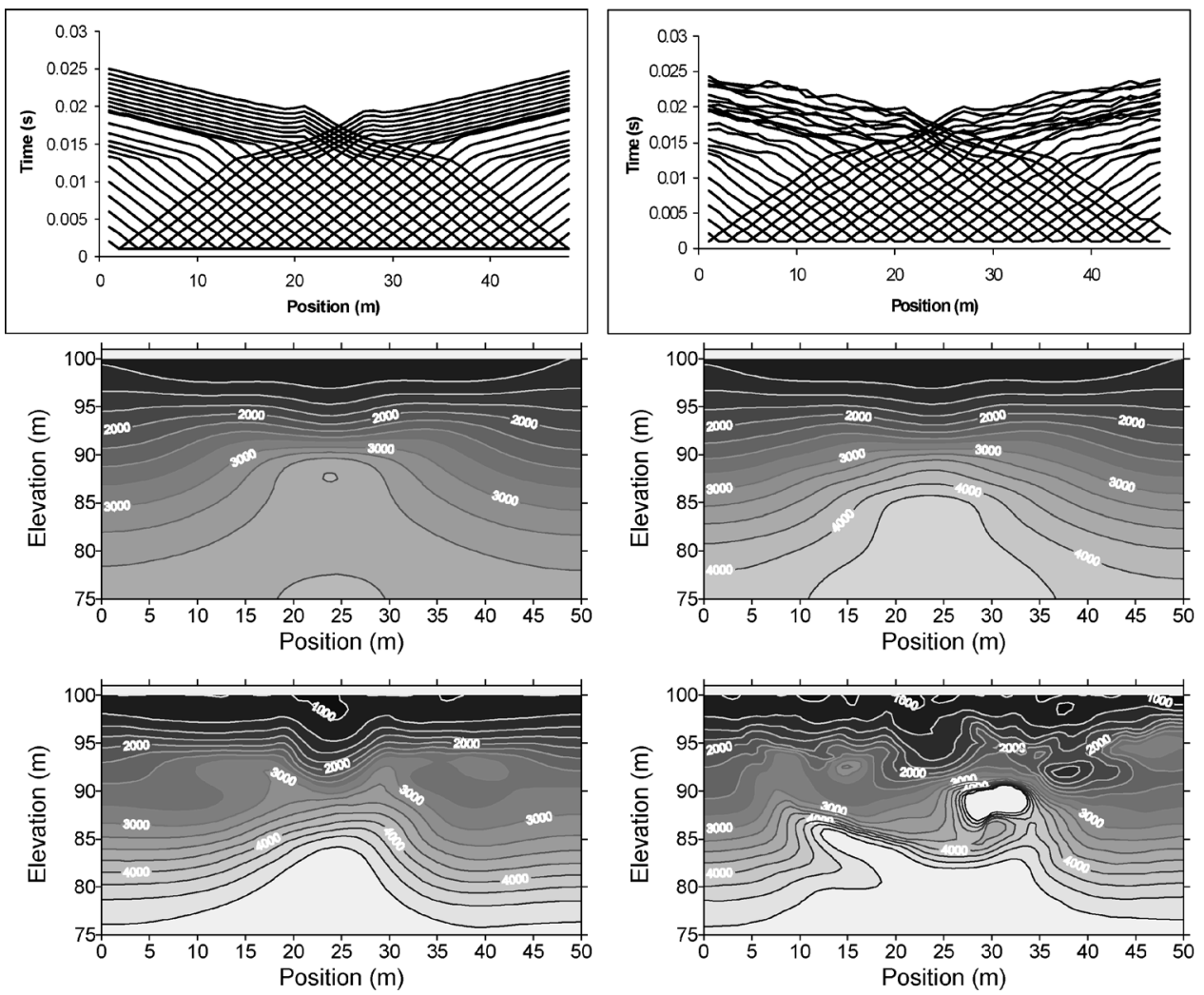

Figure 22. Relationship between noise and smoothing. The top row is the travel time files used to generate the results in the next two rows. The middle row is with normal smoothing. The bottom row is with very low smoothing. Plots show velocity in $\mathrm{m} / \mathrm{s}$.

SeisImager is very straightforward to use and only requires that the data be loaded and an initial model generated, which can be done in seconds. The code does not have the ability to use models from outside the code as initial models and does not allow the user control over cell size.

GeoCT-II requires more user initiation time because each step of the process is done in a separate subroutine. It offers excellent flexibility because you can generate any kind of initial model using the model builder. It also runs faster than the other two codes.

\section{Conclusions}

Although we have compared three codes throughout this paper, it is critical to address the performance of refraction tomography in general, compared to conventional methods. The conventional methods, such as the delay-time, or the generalized reciprocal method, have been used effectively to address a large number of near surface problems. Where they have fallen short, it is because the site conditions conflicted with fundamental assumptions of the methodology, i.e., laterally continuous constant velocity layers.
Refraction tomography methods will similarly fail when they encounter site conditions that conflict with the underlying assumptions of these methods. Models 1 and 4, for instance, show conditions that would best be addressed with conventional methods. The tomography methods insert a gradient where none is present.

Our results serve to validate all three of the codes that we compared. Each has its own strengths and weaknesses, but in the end, the average performance is similar. Although we have not aimed at achieving such a balanced result, we have found that the authors of all three codes have been extremely responsive to our questions and concerns during the two year period of this study, and have made many improvements during that time frame that have contributed to their similar performance in this comparison. We anticipate that they will continue to improve as more practitioners become familiar with the strengths and benefits of these methods.

The results of this study also provide evidence of pitfalls that users should recognize. First, it is critically important to anticipate artifacts that may be caused by lack of ray coverage. Second, it is important to have enough smoothing to eliminate artifacts, but also to avoid oversmoothing. Modeling should be used to assess the reliability 
Journal of Environmental and Engineering Geophysics

of any field result. The "narrow epikarst" model (Model 7) demonstrates that there are limits to what one can expect from a refraction tomography code.

With respect to karst applications, we have demonstrated that refraction tomography can address many of the features observed in these environments. It is also important to consider the three-dimensional character of karst terrains. All of the currently-available commercial products assume two-dimensional structures. Although superior to conventional methods for many aspects of karst terrains, they will fail where structures underlying the profile are highly threedimensional. We look forward to the next generation of software that will enable resolution of even more complicated models.

\section{Acknowledgments}

Oak Ridge National Laboratory is managed by UT-Battelle, LLC for the U. S. Department of Energy under contract DE-AC0500OR22725. The submitted manuscript has been authored by a contractor of the U. S. Government. Accordingly, the U. S. Government retains a nonexclusive, royalty-free license to publish or reproduce the published form of this contribution, or allow others to do so, for U. S. Government purposes. We would like to thank the anonymous reviewer for his/her helpful comments and suggestions.

\section{References}

Geometrics inc. and OYO, inc., 2003, SeisImager manual, version 3.
Gebrande, H., and Miller, H., 1985, Refraktionsseismik, in Angewandte Geowissenschaften II. Bender F. (ed.). Ferdinand Enke, Stuttgart, 226-260.

Haeni, F., Grantham, D., and Ellefsen, K., 1987, Microcomputerbased version of SIPT; a program for the interpretation of seismic-refraction data. U.S. Geological Survey Open-File Reports: 87-103-A,B.

Lankston, R.W., (1990) High-resolution refraction seismic data acquisition and interpretation: Geotechnical and Environmental Geophysics, Society of Exploration Geophysicists, 45-73.

Palmer, D., 1980, The generalized reciprocal method of seismic refraction interpretation. Society of Exploration Geophysicists, Tulsa.

Rohdewald, S.R., 1999, Rayfract manual.

Rohdewald, S.R., (in preperation), Delta-t-V 1D seismic refraction inversion method: Theory.

Schuster, G.T., and Quintus-Bosz, A., 1993, Wavepath eikonal traveltime inversion: theory: Geophysics, 58(9), 1314-1323.

Sheehan, J.R., Doll, W.E., and Mandell, W., 2003, Evaluation of refraction tomography codes for near-surface applications: Extended abstract: Presented at the 2003 Annual Meeting of the Society of Exploration Geophysicists, Dallas, Texas, October 26-31, 2003, 4 pp.

Watanabe, T., Matsuoka, T., and Ashida, Y., 1999, Seismic traveltime tomography using Fresnel volume approach: Expanded Abstracts. 69 ${ }^{\text {th }}$ Ann. Internat. Mtg., Society of Exploration Geophysics.

Woodward, M.J., and Rocca, F., 1989, Wave-equation tomography: Expanded Abstracts: $58^{\text {th }}$ Ann. Internat. Mtg., Society of Exploration Geophysics, 1232-1235.

Zhang, J., and Toksoz, M., 1998, Nonlinear refraction traveltime tomography. Geophysics, 63(5), 1726-1737. 